\title{
Psoriatic arthropathy and seronegative rheumatoid arthritis with psoriasis: distinguishing features from Reiter's syndrome ${ }^{1}$
}

\author{
V. WRIGHT \\ From the Rheumatism Research Unit, Leeds
}

In dealing with this subject I feel like the man who, when asked directions to a certain town, replied: 'If I were you I wouldn't start from here.'

We have previously reported 11 patients (nine men, two women) with Reiter's syndrome (RS) who went on to develop psoriatic arthritis (PSA). ${ }^{337}$ Their average age was 34 years (range 24-59). Five had multiple attacks, three had one attack, and two had prostatitis. Four had iritis and three had conjunctivitis. Sacroiliitis was present in nine. This overlap, in which one disease shades into another, has been confirmed by others. ${ }^{159}, 181,215,246$ The clinical and histological features of pustular psoriasis may be indistinguishable from those of keratodermia blennorrhagica. Indeed, when I asked our dermatology department for material to make the distinction implied in the title of this paper they were unable to find anything conclusive in their extensive pictorial collection. Morris Ziff, of Dallas, has a fascinating case of a man who developed severe RS who was ultimately treated with 6-mercaptopurine with benefit. The gross skin lesions gradually subsided and he was left with what appeared to be PSA. Gradually the 6-mercaptopurine was withdrawn. The condition then recurred, but once again as fully-fledged RS (Ziff, M., personal communication). The family studies of John Lawrence are also of interest in this context. He found that probands with RS had an increased prevalence of psoriasis among the relatives as well as ankylosing spondylitis.

I shall not deal with the role of HLA-B27, since that features elsewhere in the programme. There are, however, features that it is of interest to contrast. The sex ratio is different in the two diseases. Venereal RS affects 20 men to one woman. Dysenteric RS 10 men to one woman, and our study of

\footnotetext{
1 Together with comments by Professor W. Martel, of the University Hospital, Michigan, USA, on the radiological differential diagnostic problems presented by psoriatic arthritis, ankylosing spondylitis, and Reiter's syndrome.
}

childhood RS showed that the ratio was reduced to five boys to one girl. ${ }^{165}$ Our figures for PSA show a male :female sex ratio of $0 \cdot 7: 1$. Our analysis of age at onset shows that RS has a peak age at onset of between 26 and 35 years, while that of PSA tends to begin later in life (Fig. 1).

At the onset of the arthritis the ankle is more often affected in RS than in uncomplicated PSA, while fingers and toes are more often affected in PSA than in RS (Fig. 2). Certainly a dactylitis of the fingers is more often associated with PSA than with RS. The onset is also more often acute with fever in RS. ${ }^{335 a}$

The joints that bear the brunt of the attack in RS are the knee, the ankle, the hip, the lumbar spine
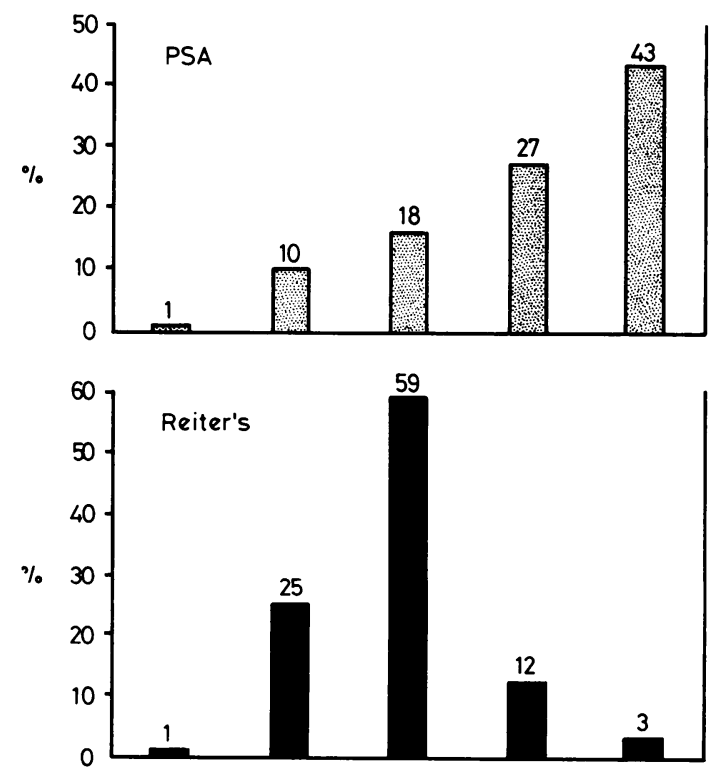

Fig. 1 Age at onset (years) in psoriatic arthritis (PSA) and Reiter's syndrome. 


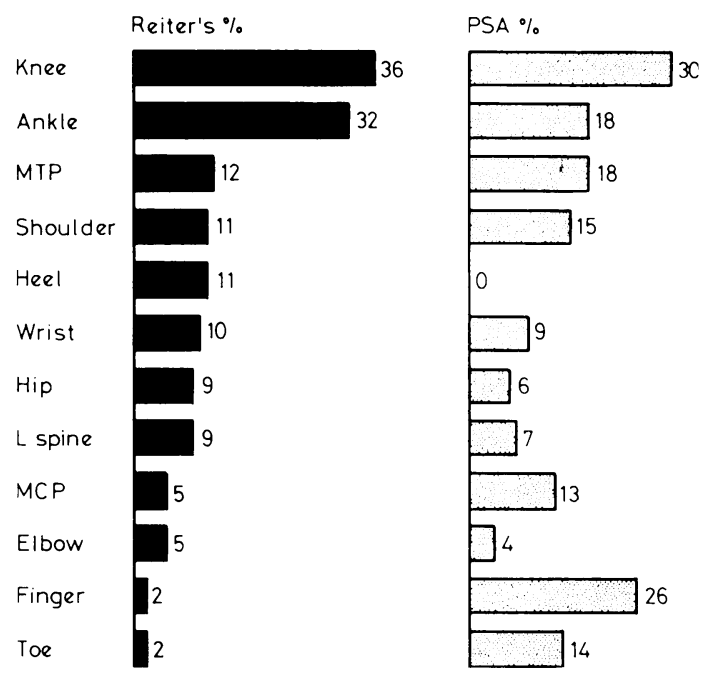

Fig. 2 Incidence ( $\%$ of patients) of joints involved at onset in PSA and RS.

and the heel. In PSA, however, the metatarsophalangeal, the fingers, the metacarpophalangeal joints, and the cervical spine are the joints more often affected (Fig. 3).

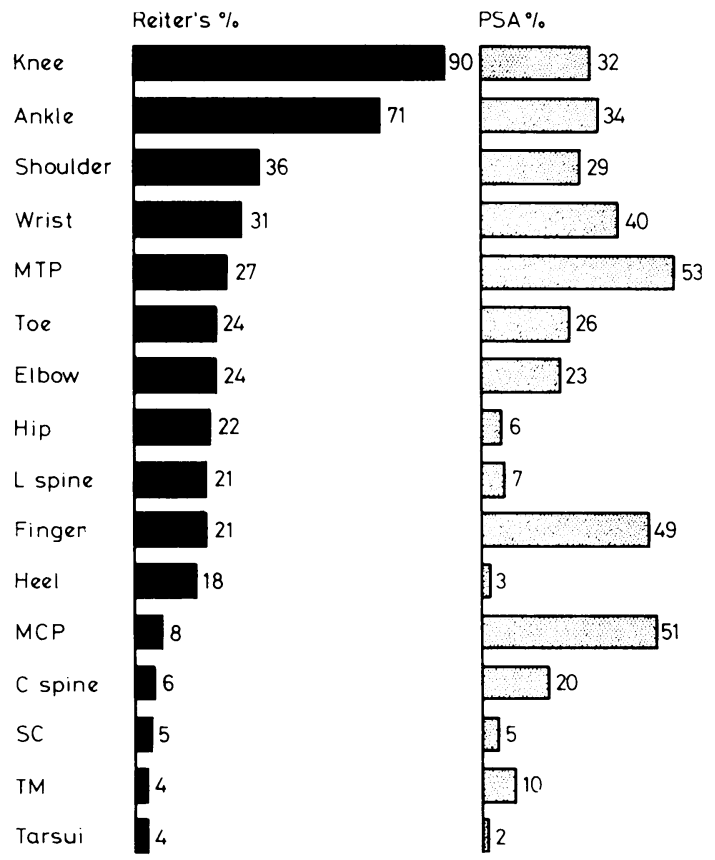

Fig. 3 Pattern ( $\%$ of patients) of joint involvement in PSA and RS.
Keratodermia blennorrhagica in RS usually begins on the soles and may affect the palms. In PSA, of course, these sites are not usually affected except in pustular psoriasis. The common sites are the elbows, knees, scalp, and trunk. Psoriasis commonly antedates the arthritis in PSA, while keratodermia blennorrhagica rarely precedes the arthritis in RS.

In a recent study of psoriatic nail dystrophy ${ }^{96}$ we found that onycholysis alone, in the absence of previous injury to the affected nail, is in favour of a psoriatic origin. A combination of at least two of onycholysis, horizontal ridging, and nail pitting present in the same patient also argues in favour of a psoriatic origin. The presence or absence of nail pitting alone is a poor discriminator between PSA and other causes of nail dystrophy, but over 20 pits per person is suggestive of a psoriatic cause of the dystrophy. Over 60 pits per person is unlikely to be found in the absence of psoriasis. The nail lesions of RS are a gross dystrophy affecting the whole nail plate.

Circinate balanitis is a feature of RS, but psoriatic lesions on the penis may cause similar appearances, although rare in PSA. Mouth ulcers and urethritio are similarly rare in PSA. Eye lesions, however, are more common in PSA than has previously beer thought. ${ }^{194}$ Iritis occurs in $3.5 \%$ of cases, rising to $\vec{\sigma}$ $10.5 \%$ in patients with psoriatic spondylitis, and conjunctivitis occurs in $21.0 \%$. The incidence is smaller in PSA than in RS, but it definitely occurs. RS patients usually have a few visual disabilities after six months and they are quite rare after 12 months, in contrast to PSA.

A high erythrocyte sedimentation rate (ESR) is much more a feature of RS than of PSA (Fig. 4).

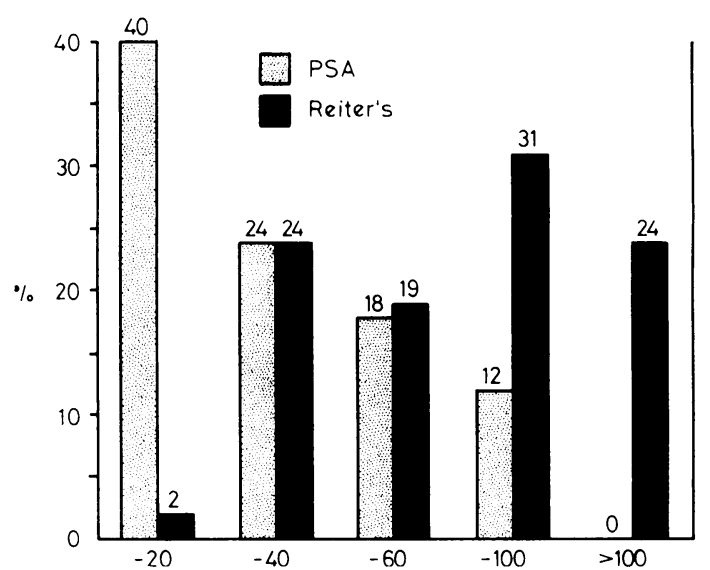

Fig. 4 Erythrocyte sedimentation rate $(\mathrm{mm}$ in $1 \mathrm{~h})$ in patients $(\%)$ with PSA or RS. 
Rheumatoid factor is absent from the serum in both groups of patients. HLA-B27 is found in $73 \%$ of RS patients, in $64 \%$ of PSA patients who have spondylitis, and in $32 \%$ of PSA patients with a distal joint form of the arthritis. ${ }^{231}$

\section{Comments on radiological diagnostic problems by Professor W. Martel}

PROF. MARTEL: I agree with many of Professor Wright's points. The following cases illustrate some of the radiological diagnostic problems presented by the overlap between psoriatic arthritis (PSA), ankylosing spondylitis (AS), and Reiter's syndrome (RS).

The first patient (Fig. 1) is the type to which Prof. Wright referred. He is B27-positive and has psoriasis and classic AS. The changes in his lumbar spine are identical to those in AS and the cervical and dorsal segments were completely involved. There were no 'skipped' segments. The second patient (Fig. 2), on the other hand, with psoriasis and peripheral arthritis, has sacroiliac joint involvement which has remained unilateral over a period of several years. In my experience, such unilaterality is extremely rare in AS, if it occurs at all. Incidentally, I prefer to call such joint disease sacroiliac arthritis and not 'sacroiliitis', because this inflammation is primarily in the joint and only secondarily affects the bone.

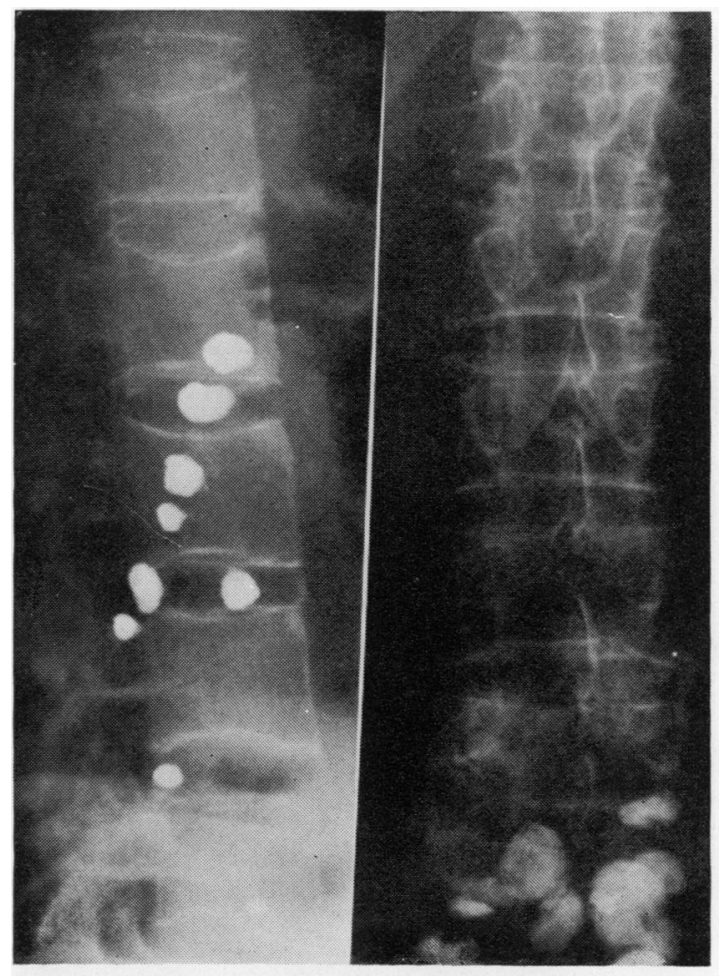

(b)

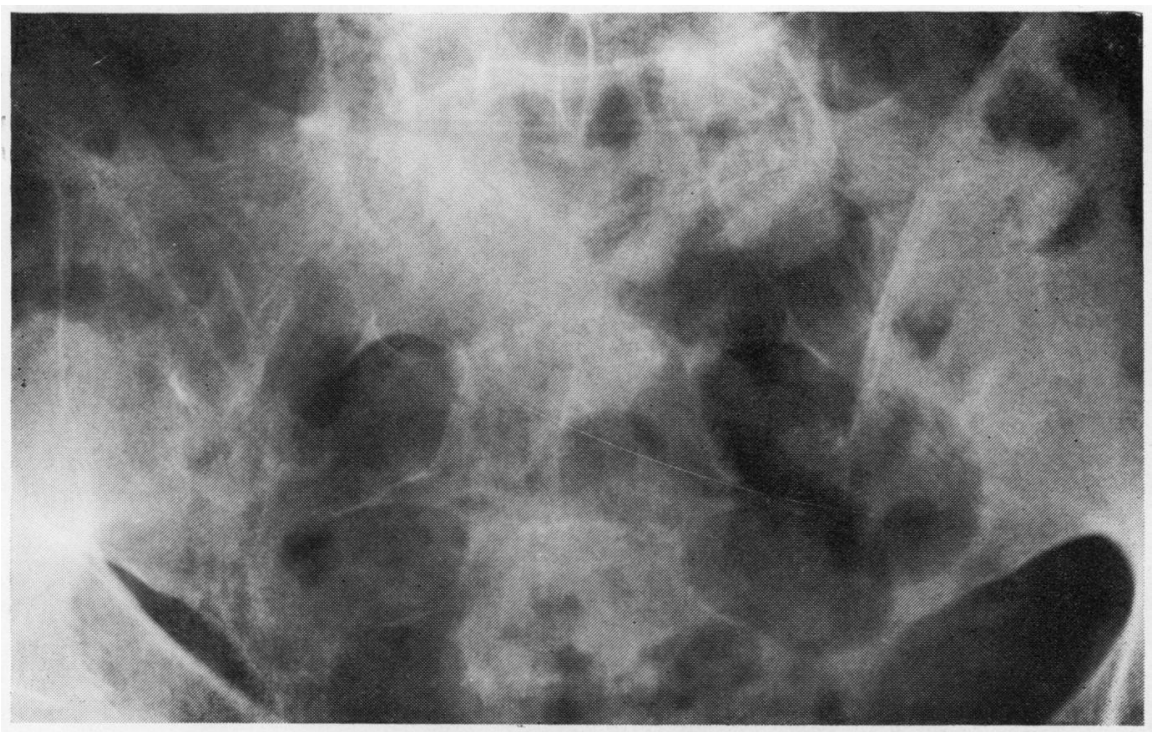

Fig. $1 A, B, C, D$. Ankylosing spondylitis in $H L A-B 27-p o s i t i v e$ patient with psoriasis. ( $C, D$ next page.)

(a) 
(d)

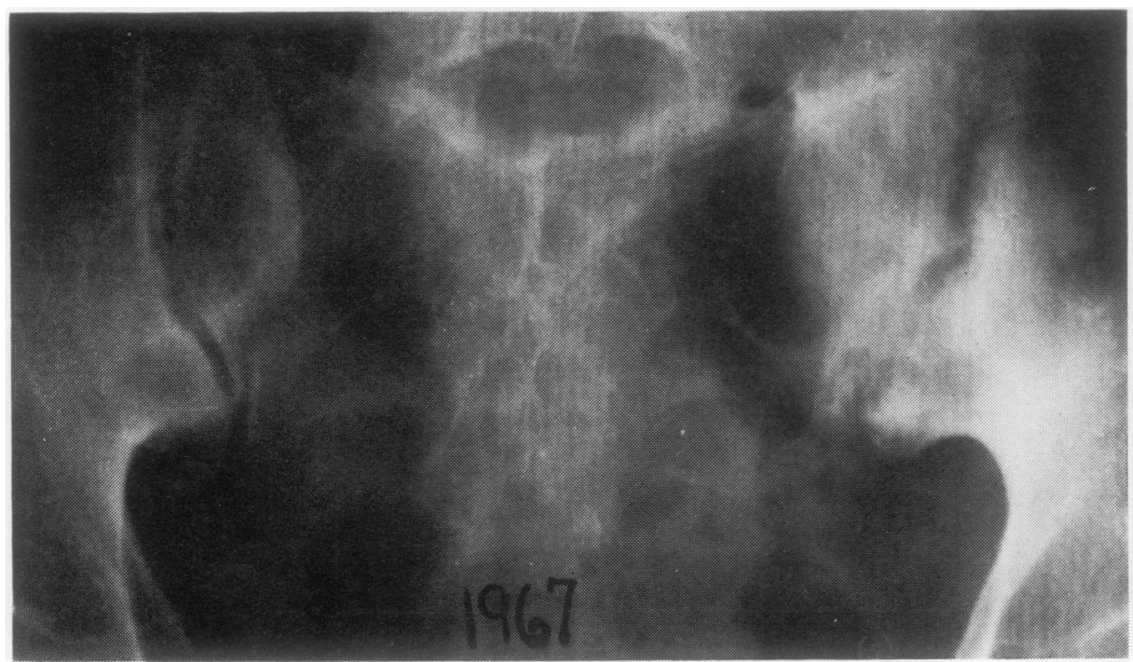

Fig. 2 Unilateral sacroiliac arthritis in patient with psoriasis and peripheral arthritis. 
Fig. 3 shows the lumbar spine of a patient with classic PSA of the peripheral joints. There is heavy bony bridging restricted almost exclusively to the left side of the lumbar spine, similar to that seen in RS.

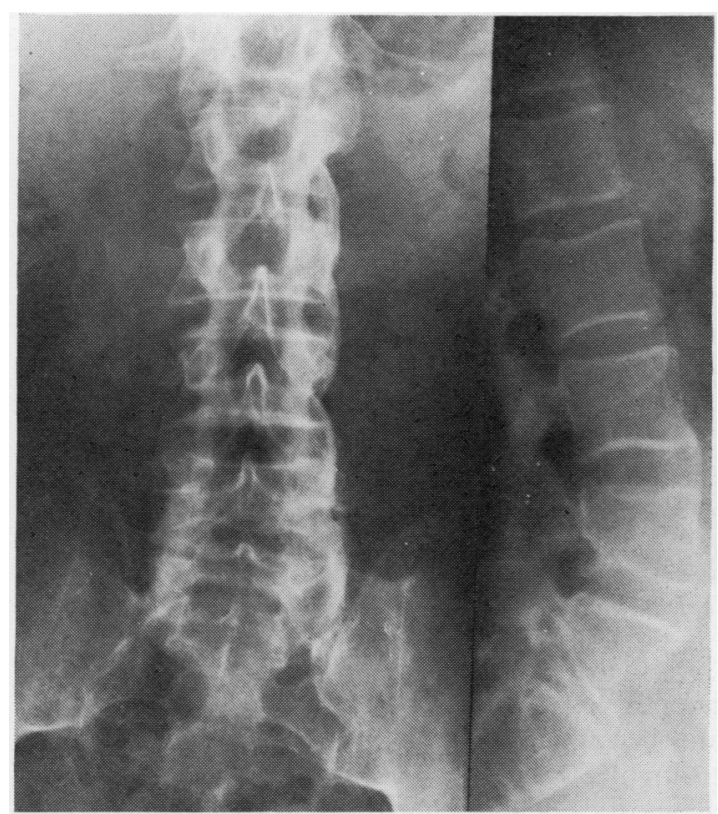

Fig. 3 Lumbar spine of patient with psoriatic arthritis of peripheral joints. Note bony bridging, mostly left.
Fig. 4 shows a characteristic, peculiar ossification. Its location and configuration suggest a relation to the lateral longitudinal ligament in the dorsolumbar spine. This was described by Bywaters and Dixon ${ }^{49}$ in four patients with psoriatic arthritis. In these cases the anterior ligaments appears to be spared. We have seen this in PSA and in RS. I have seen one patient with PSA with a similar appearance except that the linear ossification was discontinuous but, as in this case, unrelated to the vertebrae.

Calcaneal lesions consisting of extensive erosion, bone proliferation, and sclerosis (Fig. 5) may be seen both in PSA or RS. I've not seen such lesions in rheumatoid arthritis and rarely does one see this kind of extensive destruction and bone apposition in AS.

A tendency for the interphalangeal joint of the great toe to be affected in RS has been referred to. Fig. 6 shows an identical appearance in a patient with PSA. Occasionally in PSA there is resorption of the terminal tuft of the large toe associated with extensive sclerosis of this phalanx, as shown in Fig. 7. I have not yet seen this sclerosis in RS but we are keeping an open mind as to its specificity. It may well be a unique feature of PSA.

The next case (Fig. 8) exhibits two features of PSA which I believe are diagnostically significant. The first is a tendency for involvement of multiple joints all in the same hand, with sparing of all the joints in the other hand. The second feature is a
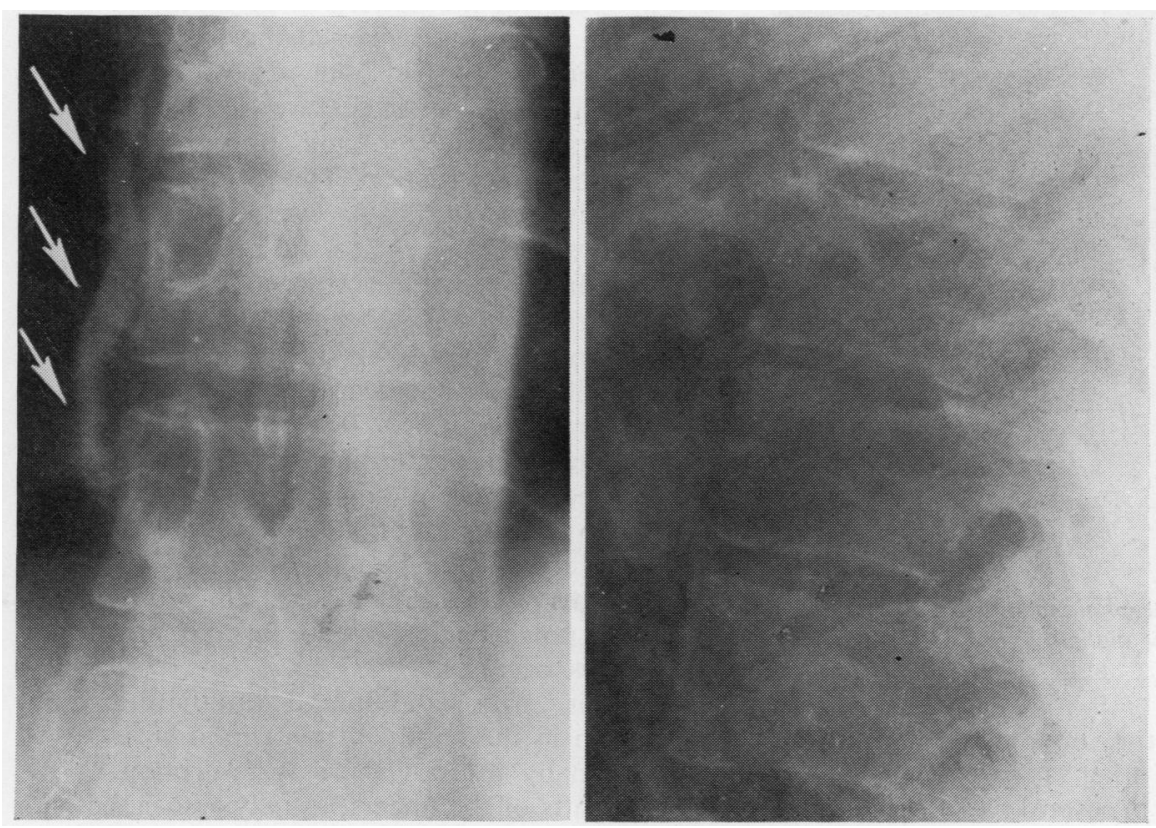

Fig. 4 Dorsolumbar spine of patient with psoriatic arthritis showing (arrows) characteristic ossification apparently related to lateral longitudinal ligament. 


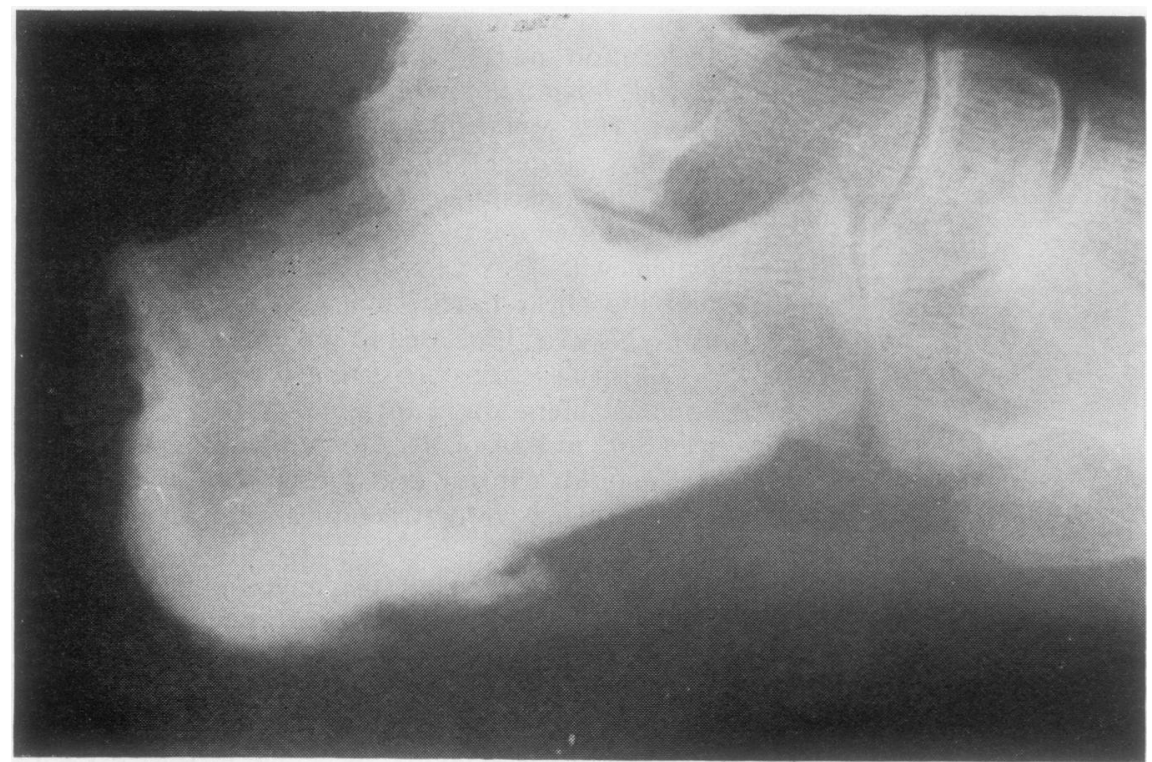

Fig. 5 Extensive erosion, bone proliferation, and sclerosis of calcaneum as seen in psoriatic arthritis and RS.

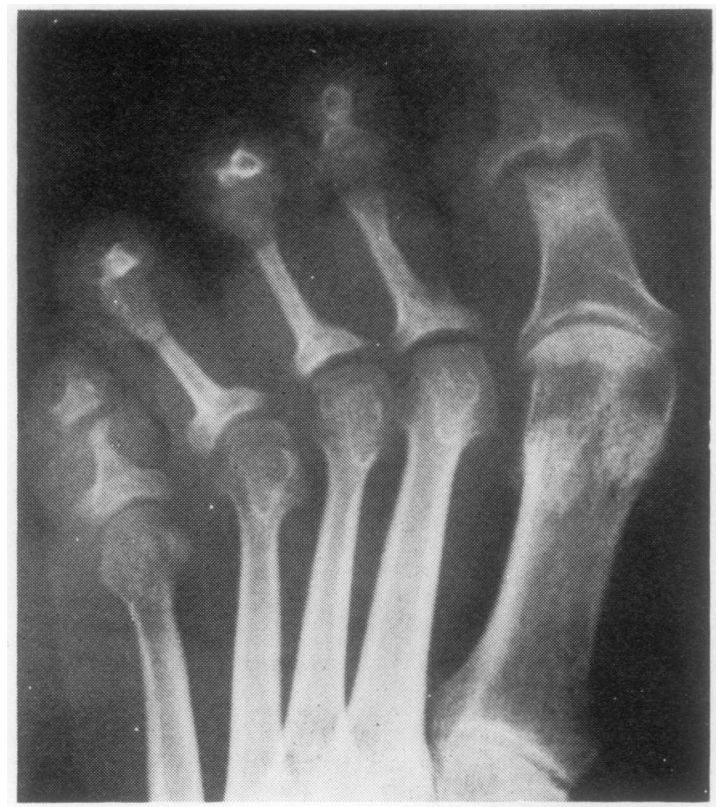

Fig. 6 Involvement of interphalangeal joint of great toe in patient with psoriatic arthritis.

tendency for joints of the same ray to be affected whereas all the joints of other rays are conspicuously spared. I have seen this in several patients with PSA but not yet in RS. This patient has extensive exuber ant periosteal bone apposition, but the tendency to involve the joints of a single ray can be useful diagnostically, particularly in those cases such as this one where there is no bone apposition. I have seen a patient who had progressive joint destruction over several years but the pattern of involvement of the joints of a single ray remained the same and there was not much periosteal bone apposition.

Fig. 9 shows a classic feature of PSA-namely, involvement of the terminal finger joints with extensive bone destruction. This could be confused with erosive osteoarthritis except for the fluffy bone apposition adjacent to the sites of bone destruction, the tendency for erosions to appear at the marginal rather than subchondral areas, and the involvement of virtually all the joints of the wrists. Occasionally a terminal interphalangeal joint is involved in patients with RS, but I have not seen involvement of all the terminal joints in RS.

The patient whose radiograph is shown in Fig. 9 went on to develop a mutilating arthritis of virtually all the joints of the hands and wrists (Fig. 10). I believe this raises another useful differential point. Although polyarticular disease of the hands and wrists does occur in RS, I have never seen such panarthritis with widespread mutilating joint destruction in the hands in this condition.

Occasionally in PSA there is resorption of the terminal tufts of the fingers, usually associated with 


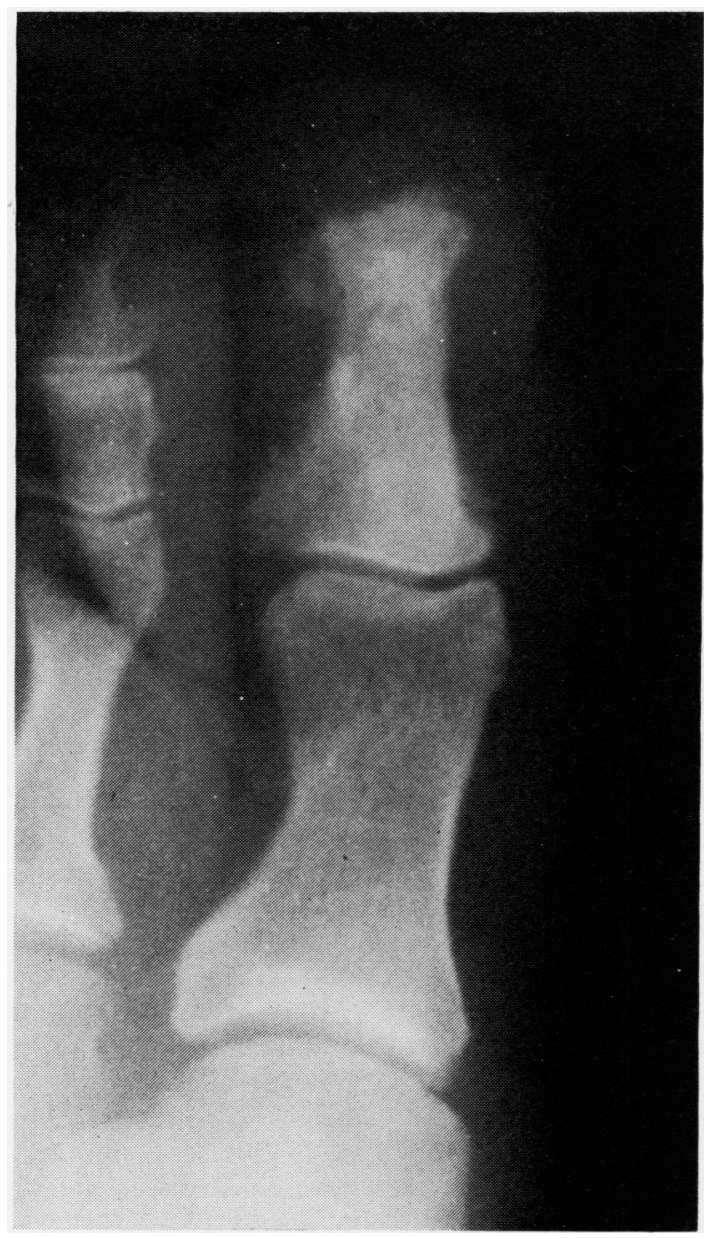

Fig. 7 Resorption of terminal tuft of great toe with sclerosis of phalanx in patient with psoriatic arthritis.

involvement of the adjacent terminal interphalangeal joints, as seen in Fig. 11. There may be soft tissue swelling around the terminal phalanx. I've not seen such terminal tuft resorption in the fingers in RS.

My position on the radiological differential diagnosis of PSA is as follows. I believe that there is a significant overlap, particularly between RS and PSA and, to a lesser extent, between these two conditions and AS, but there are significant differences. This is a summary of a study that Dr Armand Good and I completed 12 years ago comparing RS and PSA.

We presented these observations at the annual meeting of the Radiologic Society of North America in 1968. Table 1 addresses itself to the sacroiliac
Table 1 Comparison between sacroiliac disease in 26 patients with Reiter's disease (RS) and in 14 patients with psoriatic arthritis (PSA)

\begin{tabular}{lll}
\hline Sacroiliac joints & $R S(n=26)$ & $P S A(n=14)$ \\
\hline Symmetrical arthritis & 15 & 8 \\
Asymmetrical arthritis & 7 & 3 \\
Unilateral & 3 & 2 \\
Sclerotic 'patch' & 4 & 0 \\
None & 4 & 3 \\
\hline
\end{tabular}

joints in these two conditions. There were 26 patients with RS and 14 with PSA. The prevalence of both symmetrical and asymmetrical sacroiliac arthritis was almost the same in both groups. There were five patients with unilateral sacroiliac arthritis, three with RS, and two with PSA. A patch of sclerosis above the sacroiliac joint was observed in four patients with RS. This lesion might be a 'sacroiliitis' because it doesn't involve the joint proper. It may truly represent an osteitis and periostitis.

Table 2 shows the vertebral changes in these two groups of patients. It is of interest that features indistinguishable from AS were seen in three patients, two with RS and one with PSA. It was much more common to see a spondylitis which was atypical for AS either because there were large asymmetric bony bridges between vertebral bodies with sparing of the anterior compartment or skipped segments such as we described earlier.

We have been looking at these so-called large asymmetrical syndesmophytes which, incidentally, are indistinguishable in RS and PSA but are different from the syndesmophytes of AS. The latter have been termed marginal, and I suspect it is because they tend to occur at the discovertebral margins. Those in RS and PSA are different in that they are broad-based and are situated more posteriorly than those in AS. They may in fact primarily represent a periostitis of the vertebral bodies with secondary bone proliferation, and in this sense they may differ in pathogenesis from the syndesmophytes of AS.

Table 2 Comparison between radiological vertebral changes in the same groups of patients as in Table 1

\begin{tabular}{lcl}
\hline Spondylitis & $R S(n=26)$ & $P S A(n=14)$ \\
\hline Typical for AS & 2 & 1 \\
Atypical for AS & 11 & 5 \\
$\quad$ Large asymm. syndesmo. & 7 & 3 \\
$\quad$ 'Skipped' segments & 6 & 3 \\
None & 12 & 8 \\
\hline
\end{tabular}

AS = ankylosing spondylitis. 


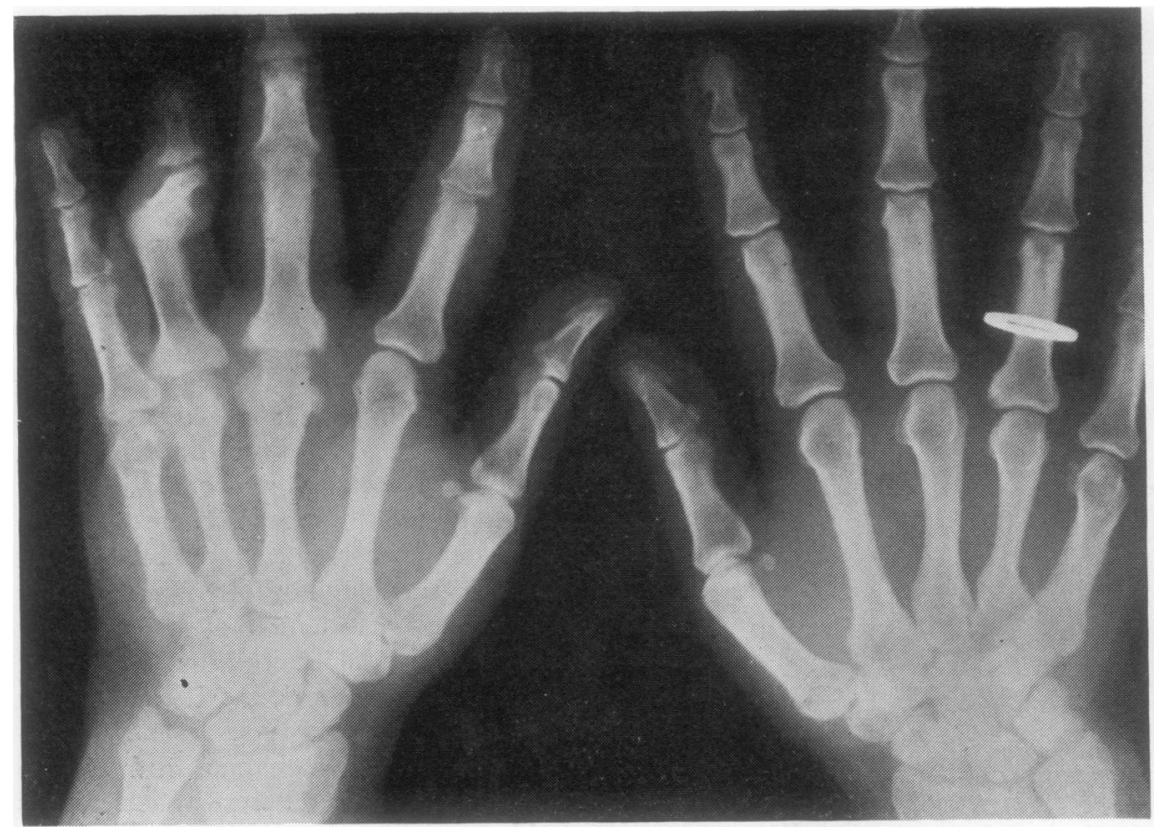

Fig. 8 Hands of patient with psoriatic arthritis. Note joints of only one hand affected and joints of same ray affected while joints of other rays spared.

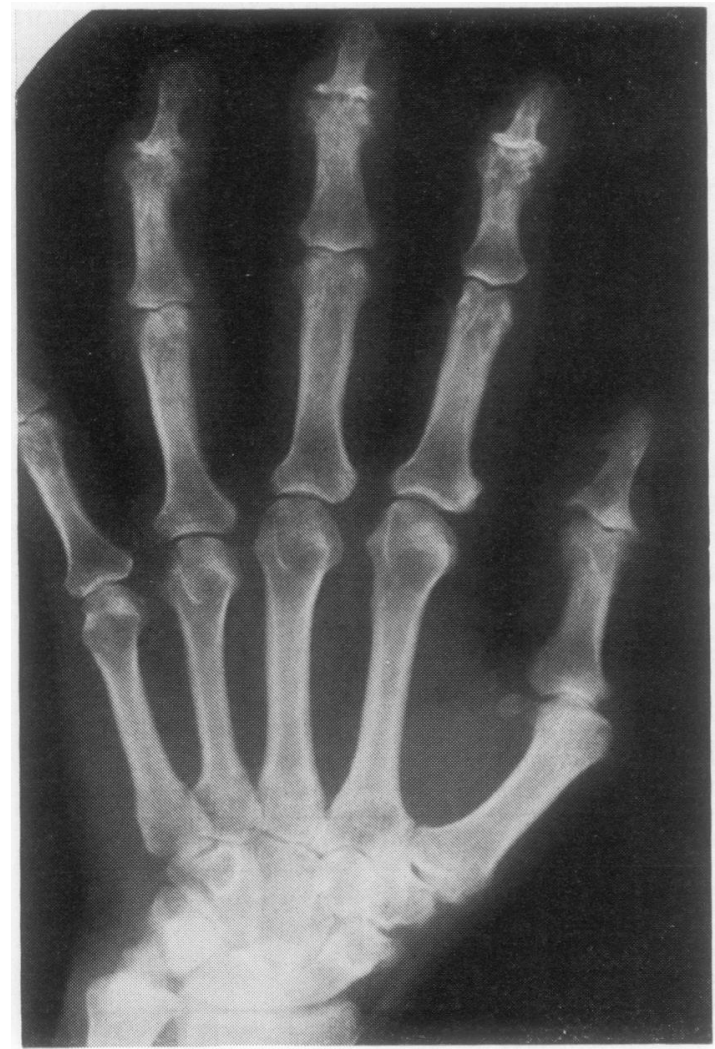

Table 3 shows the pattern of peripheral joint involvement in these two conditions. Periostea bone apposition is common in both groups but terminal interphalangeal joint involvement of the hands is a feature typical of PSA. It does not occur in every case of psoriasis but it is uncommon in RS. Interphalangeal joint involvement of the great toe occurs in both conditions, although in this series it seemed to be more common in PSA. It is of interest that arthritis may be more severe in the feet than in the hands not only in RS but also in PSA.

Table 3 Comparison between patterns of peripheral joint changes in the same groups of patients as in Tables 1 and 2

\begin{tabular}{lll}
\hline Peripheral skeletal changes & $R S(n=26)$ & $P S A(n=14)$ \\
\hline Periosteal bone apposition & 19 & 9 \\
Term. interphal. joints, & 1 & 10 \\
$\quad$ hands & 4 & 10 \\
Interphal. joint, gr. toe & 8 & 4 \\
Severity: & 4 & 1 \\
$\quad$ Feet $>$ hands & 11 & 7 \\
Feet $>$ hands & 5 & 2 \\
Heel involvement: & & \\
Slight to Moderate & Severe &
\end{tabular}

Fig. 9 Arthritis of terminal finger joints with extensive bone destruction: classic feature of psoriatic arthritis. 


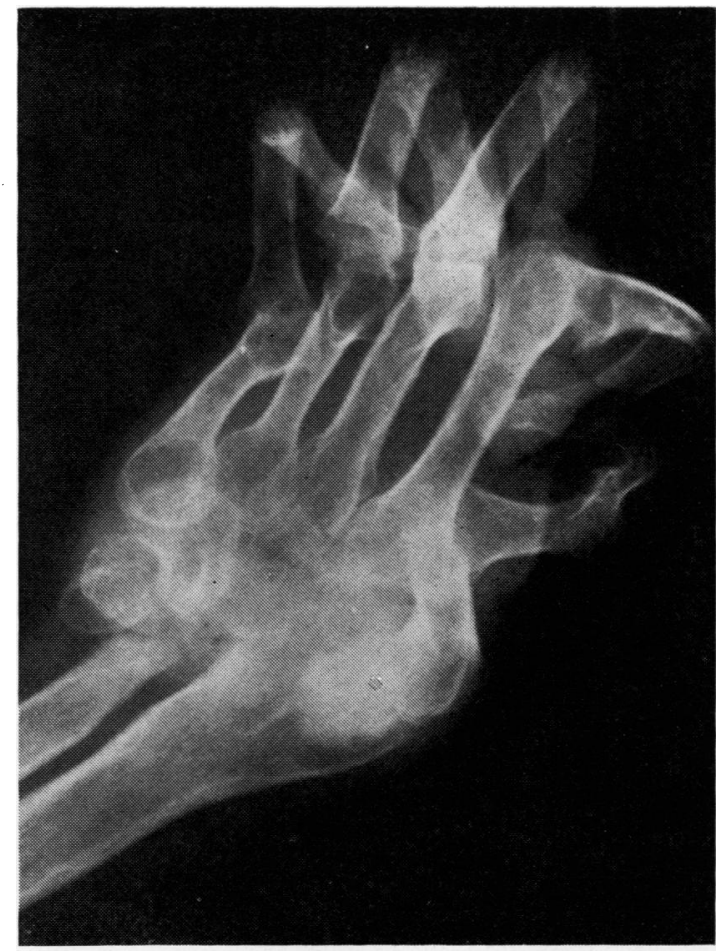

Fig. 10 Mutilating arthritis of joints of hand and wrist: a later development in patient shown in Fig. 9.

\section{General discussion}

PROF. M. ZIFF: What do you do when you see this kind of $x$-ray change and the patient has neither psoriasis or Reiter's? It happens does it not?

PROF. MARTEL: Yes, but it is very uncommon. A significant number, about $10 \%$ of patients with PSA, start off with joint disease and the psoriatic lesions come later. The important thing is to take note of these clinically atypical as well as radiologically atypical cases and evaluate them again later. PROF. A. E. GOOD: Professor Wright, did you have a chance to sort out your overlaps on the basis of the $B$ antigens 13,17 , and 38 ?

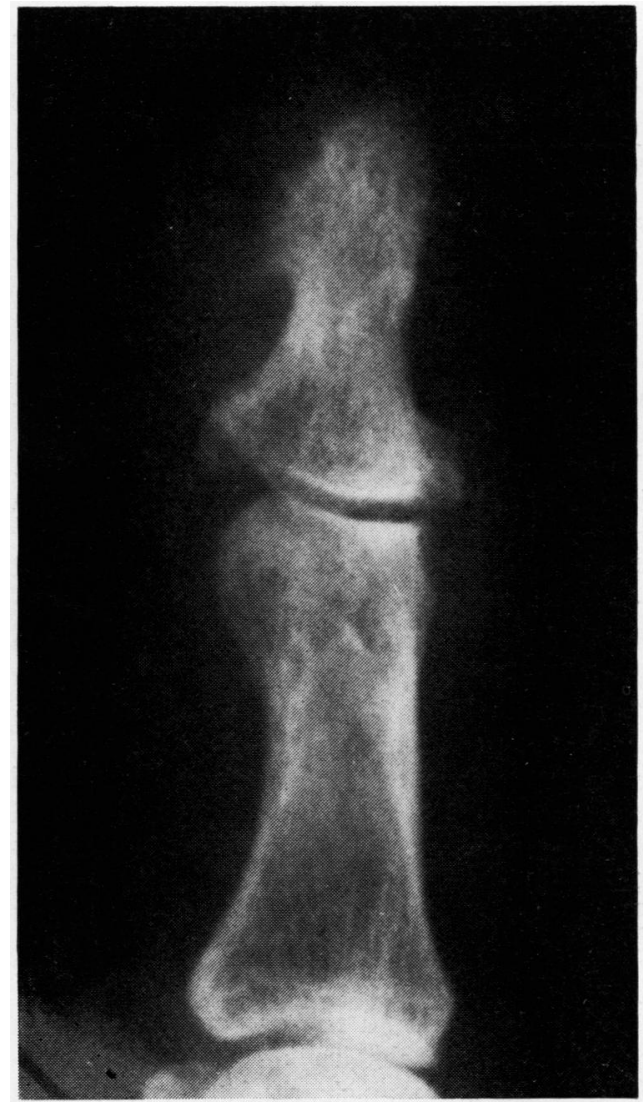

Fig. 11 Resorption of terminal tuft of finger and arthritis of terminal interphalangeal joint in patient with psoriatic arthritis.

PROF. WRIGHT: No.

DR. J. C. WOODROw: Mention was made of the group of patients who start with RS and go on to get skin lesions of psoriasis and corresponding joint lesions. All such patients I have seen were B27positive. Has anybody seen this syndrome in a B27negative patients? It looks as though B27 is playing a part in producing susceptibility to this particular disease combination. 